\title{
The massive and massless Einstein-Vlasov system with spherical symmetry
}

\author{
Peter Eigenschink and David Fajman \\ Gravitational Physics, Faculty of Physics, University of Vienna, Boltzmanngasse 5, 1090 Wien, Austria
}

Jérémie Joudioux

Department of Mathematics, Faculty of Science, Radboud University, PO Box 9010, Postvak 59, 6500 GL Nijmegen, Netherlands

(Received 11 May 2018; published 1 August 2018)

\begin{abstract}
We analyze the asymptotic behavior of small perturbations of Minkowski space caused by the presence of ensembles of massive and massless particles in spherical symmetries. The perturbations we consider are compactly supported in space and momenta.
\end{abstract}

DOI: $10.1103 /$ PhysRevD.98.044002

\section{INTRODUCTION}

We address in this article the small data global existence problem for the Einstein-Vlasov system with massive and massless particles in spherical symmetry. This system models spacetime with two ensembles of particles, massive and massless, interacting solely through gravitation. Using the standard notations for the metric of the form $-e^{2 \mu} d t^{2}+e^{2 \lambda} d r^{2}+r^{2} d \Omega^{2}$ (see [1]), the system then is

$$
\begin{aligned}
e^{-2 \lambda}\left(2 r \lambda^{\prime}-1\right)+1 & =8 \pi r^{2} \rho, \\
e^{-2 \lambda}\left(2 r \mu^{\prime}+1\right)-1 & =8 \pi r^{2} p, \\
\dot{\lambda} & =-4 \pi r e^{\lambda+\mu} j, \\
e^{-2 \lambda}\left(\mu^{\prime \prime}+\left(\mu^{\prime}-\lambda^{\prime}\right)\left(\mu^{\prime}+r^{-1}\right)\right)-e^{-2 \mu}(\ddot{\lambda}+\dot{\lambda}(\dot{\lambda}-\dot{\mu})) & =4 \pi q, \\
\rho(t, x) & =\int \sqrt{1+|\mathrm{v}|^{2}} f^{m v}(t, x, \mathrm{v}) d \mathrm{v}+\int|\mathrm{v}| f^{m l}(t, x, \mathrm{v}) d \mathrm{v}, \\
p(t, x) & =\int\left(\frac{x \cdot \mathrm{v}}{r}\right)^{2} f^{m v}(t, x, \mathrm{v}) \frac{d \mathrm{v}}{\sqrt{1+|\mathrm{v}|^{2}}}+\int\left(\frac{x \cdot \mathrm{v}}{r}\right)^{2} f^{m l}(t, x, \mathrm{v}) \frac{d \mathrm{v}}{|\mathrm{v}|}, \\
j(t, x) & =\int \frac{x \cdot \mathrm{v}}{r} f^{m v}(t, x, \mathrm{v}) d \mathrm{v}+\int \frac{x \cdot \mathrm{v}}{r} f^{m l}(t, x, \mathrm{v}) d \mathrm{v}, \\
q(t, x) & =\int\left|\frac{x \times \mathrm{v}}{r}\right|^{2} f^{m v}(t, x, \mathrm{v}) \frac{d \mathrm{v}}{\sqrt{1+|\mathrm{v}|^{2}}}+\int\left|\frac{x \times \mathrm{v}}{r}\right|^{2} f^{m l}(t, x, \mathrm{v}) \frac{d \mathrm{v}}{|\mathrm{v}|},
\end{aligned}
$$

where $f^{m v}$ and $f^{m l}$ denote the distribution functions for massive and massless particles, respectively. We prove

Theorem I.1. Consider asymptotically flat, spherically symmetric initial data to (EVS) with compactly supported, non-negative particle distributions $f_{0}^{m v}, f_{0}^{m l} \in C^{1}\left(\mathbb{R}^{6}\right)$. There is an $\epsilon>0$ such that if

$$
\left\|f_{0}^{m}\right\|_{\infty} \leq \epsilon,
$$

then there exists a regular global in time solution $\left(f^{m v}, f^{m l}, \mu, \lambda\right)$ to (EVS) with $f^{m}(0)=f_{0}^{m}$. Moreover the maximal future development is future causally geodesically complete and the following asymptotics hold

$$
\begin{aligned}
\left\|T_{u u}\right\|_{L^{\infty}\left(C_{v}\right)} & \leq \frac{C}{(1+t)^{2}}, \\
\left\|T_{u v}\right\|_{L^{\infty}\left(C_{v}\right)}, \quad\left\|T_{v v}\right\|_{L^{\infty}\left(C_{v}\right)} & \leq \frac{C \log t}{(1+t)^{4}}
\end{aligned}
$$

in region II (a neighborhood of $\mathcal{I}^{+}$away from $i^{+}$[2]) and

$$
\|\rho\|_{L^{\infty}\left(\Sigma_{t}\right)}, \quad\|p\|_{L^{\infty}\left(\Sigma_{t}\right)}, \quad\|j\|_{L^{\infty}\left(\Sigma_{t}\right)}, \quad\|q\|_{L^{\infty}\left(\Sigma_{t}\right)} \leq \frac{C}{(1+t)^{3}}
$$


in region III (a neighborhood of $i^{+}$), with some constant $C>0$. Here $T_{u u}, T_{u v}$ and $T_{v v}$ are the projections of the energy-momentum tensor in the null directions $u$ and $v$. [3].

This theorem can be seen as a natural generalization of the corresponding results for individual types of particles established by Rein-Rendall [4] and Dafermos [5], respectively. Moreover, it can be seen as a guideline on how to address the related problem without symmetry assumptions, where the individual cases for massive and massless particles, respectively, have recently been resolved [6-8].

\section{A. Overview}

We first prove that the spatial support of the densities for massive and massless particles becomes disjoint, and more specifically are separated by a sphere. This is a consequence of the existence of upper bounds for the support in space and velocities for both densities, as well as the existence of a lower bound for the velocities of massless particles. The proof relies on elementary manipulations of the characteristic system (see Sec. II B). We then consider a Cauchy problem for massless particles near $\mathcal{I}^{+}$, where the techniques of [5] can be readily used; see Sec. II D. Then, starting with data on a hyperboloidal slice, it is sufficient to suitably adapt [4] to obtain global existence near $i^{+}$in Sec. II E. These two global existence results are finally joined together in Sec. II F. One of the difficulties lies in the comparison of the coordinate systems used in both Cauchy problems.

\section{EXISTENCE AND STABILITY}

\section{A. The local Cauchy problem}

First we essentially deal with region I of the spacetime, that is to say with the local Cauchy problem for (EVS). Most of the work has been performed for the massive system by Choquet-Bruhat in the 70s (see Sec. X of [9]), and was later on extended in [10] [10, Proposition 19.76] for the local existence and [10, Proposition 20.5] for the local stability. This extends naturally to the massless system (see [9], Theorem 12. 1 of [11]). The local existence for the Cauchy problem is obtained by collating these results together. This is performed in part II, Paper B, Theorem 8.25 of [12], based on the approach developed in [10]. The stability result, ensuring long time existence provided the initial data are small enough, can be found in part II, Paper B, Lemma 9.1 of [12].

The main issue at this point remains the splitting of the spacetime as illustrated in Fig. 1. So from local existence and stability together, we also establish that separation of the supports of $f^{m v}$ and $f^{m l}$ occurs after finite time and holds up until the end of the existence interval. Only then is it clear that there exists a finite time after which we can cut a region, like region I, out of the spacetime as desired and separate the remaining part of the spacetime in regions II and III.

The following proposition is stated in a way similar to Chapter 1.3, Theorem 3.1 of [1] and is a conclusion of that

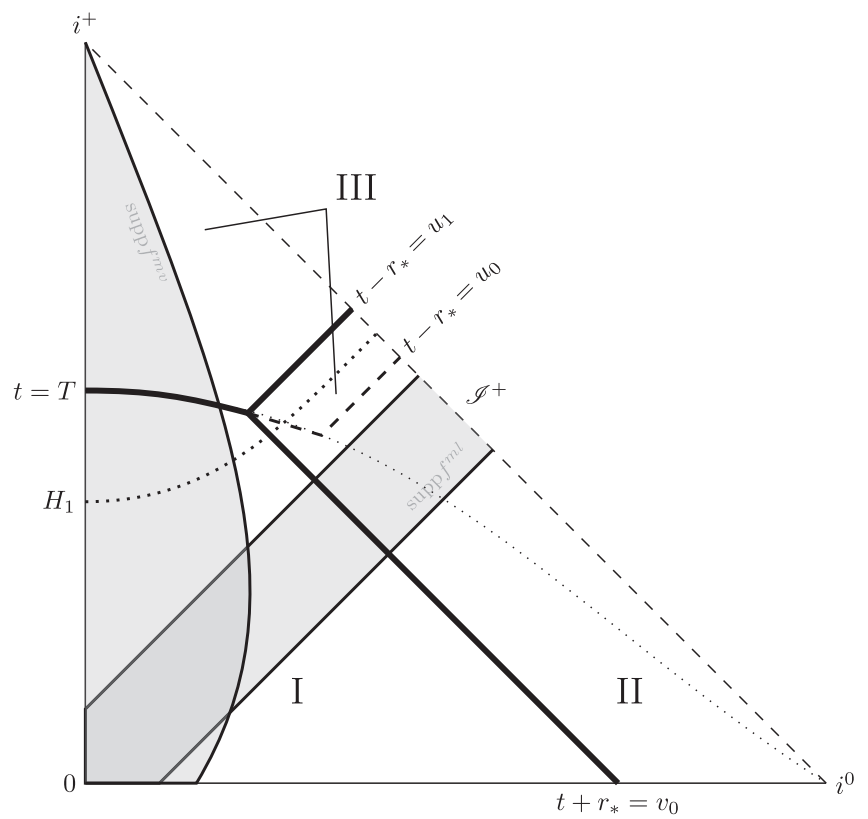

FIG. 1. The way the spacetime is sliced throughout the proof. Region I is compact and contains a combination of massive and massless particles, while region II and region III are asymptotic, containing only one type of matter each, either massive or massless. The regions are separated by the thick lines, but region III also contains the area bounded by the dashed thick line (in particular, regions II and III overlap in this part)—see Sec. II C for a detailled definition of them.

theorem together with local existence of the massless system stated in Chapter 8 of [5] and the collating of those results performed in part II, Paper B, Theorem 8.25 of [12]. It contains local existence and uniqueness of the massive-massless Einstein-Vlasov system together with a continuation criterion of that solution.

Proposition II.1. Let $f_{0}^{m v}, f_{0}^{m l} \in C^{1}\left(\mathbb{R}^{6}\right)$ be nonnegative, compactly supported and spherically symmetric. Also suppose that

$$
\int_{|x|<r} \int p_{m}^{0} f_{0}^{m} d p d x<r / 2
$$

for all $r>0$, with $p_{m v}^{0}=\sqrt{1+|p|_{2}^{2}}$ and $p_{m l}^{0}=|p|_{2}-|\cdot|_{2}$ being the standard Euclidean distance. Then there exists a unique regular spherically symmetric solution $\left(f^{m v}, f^{m l}, \lambda, \mu\right)$ to (EVS) on an interval $[0, T)$ with $f^{m}(0)=f_{0}^{m}$. Moreover, if

$$
\sup \left\{\mid p \|(t, x, p) \in \operatorname{supp} f^{m v}, t \geq 0\right\}<\infty
$$

and

$$
\begin{gathered}
\inf \left\{\mid p \|(t, x, p) \in \operatorname{supp} f^{m l}, t \geq 0\right\}>0, \\
\sup \left\{\mid p \|(t, x, p) \in \operatorname{supp} f^{m l}, t \geq 0\right\}<\infty
\end{gathered}
$$

hold, then $\mathrm{T}=\infty$. 
Remark.-The conditions (2) and (3), imply the global existence of the solution. They need to be ensured in the course of the evolution from the desired initial data. This is the continuation criterion for this system. The relation between the size of the initial perturbation and the maximal time of existence is given in the following proposition.

The Cauchy stability result stated in the next proposition is again a conclusion of statements known due to [1, Chapter 1.4] in the massive case and due to [5, Chapter 8] in the massless case, while being joint together for a combination of those particles in part II, Paper B, Lemma 9.1 of [12].

Proposition II.2. There exists $\epsilon>0$, a positive, strictly monotonically increasing function $\zeta \in C([0, \infty))$ and a positive, strictly monotonically decreasing function $\tau \in$ $C((0, \epsilon))$ such that

$$
\lim _{\beta \rightarrow 0} \tau(\beta)=\infty
$$

Then if

$$
\left\|f_{0}\right\|:=\left\|f_{0}^{m v}\right\|_{L^{\infty}\left(\mathbb{R}^{6}\right)}+\left\|f_{0}^{m l}\right\|_{L^{\infty}\left(\mathbb{R}^{6}\right)} \leq \epsilon,
$$

then

$$
T>\tau\left(\left\|f_{0}\right\|\right)
$$

Moreover, for $t \in\left[0, \tau\left(\left\|f_{0}\right\|\right)\right]$, we have the following estimate:

$$
\begin{aligned}
& \|f(t)\|+\|\lambda(t)\|_{L^{\infty}\left(\mathbb{R}^{3}\right)}+\|\mu(t)\|_{L^{\infty}\left(\mathbb{R}^{3}\right)}+\left\|e^{2 \lambda(t)}-1\right\|_{L^{\infty}\left(\mathbb{R}^{3}\right)} \\
& \quad+\|\dot{\lambda}(t)\|_{L^{\infty}\left(\mathbb{R}^{3}\right)}+\left\|\mu^{\prime}(t)\right\|_{L^{\infty}\left(\mathbb{R}^{3}\right)}+\left\|\lambda^{\prime}(t)\right\|_{L^{\infty}\left(\mathbb{R}^{3}\right)} \\
& \quad+\|\dot{\mu}(t)\|_{L^{\infty}\left(\mathbb{R}^{3}\right)}+\frac{1}{r}\left(|\dot{\lambda}|+\left|\lambda^{\prime}\right|+\left|\mu^{\prime}\right|\right)(t, r) \leq \zeta(t)\left\|f_{0}\right\| .
\end{aligned}
$$

\section{B. The separation of massive and massless particles}

Consider first that we are working in the frame of a perturbation of the Minkowski spacetime in the standard Cartesian coordinates $\left(x^{\alpha}\right)$ with

$$
g=\eta+h, \quad \Gamma(g)=\Gamma(h)
$$

with

$$
|h| \leq \epsilon \quad \text { and } \quad|\Gamma(h)| \leq \epsilon(1+t)^{-1-\alpha},
$$

for some $\alpha>0$. Here, $\eta$ is the Minkowski metric and $h$ represents a perturbation to it, with $\Gamma$ being the corresponding Christoffel symbols.

Consider a distribution of massive and massless particles such that, initially at $t=0$,

$$
\operatorname{supp}_{x} f^{m v} \subset B\left(0, R_{0}\right), \quad \operatorname{supp}_{x} f^{m l} \subset B\left(0, R_{0}\right),
$$

and

$$
\begin{aligned}
& \operatorname{supp}_{v} f^{m v} \subset B\left(0, V_{0}\right), \\
& B\left(0, V_{00}\right) \not \subset \operatorname{supp}_{v} f^{m l} \subset B\left(0, V_{0}\right) .
\end{aligned}
$$

In what follows, $|\cdot|_{2}$ is the standard Euclidean distance. We recall the mass shell relation

$$
g(v, v)=-1 \quad \text { for massive particles }
$$

and

$$
g(v, v)=0 \quad \text { for massless particles. }
$$

For either massive or massless particles, we define

$$
v_{m v}^{0}=\sqrt{1+|v|_{2}} \quad \text { and } \quad v_{m l}^{0}=|v|_{2}
$$

respectively.

Consider now massive particles. Using the identity,

$$
\frac{d r}{d t}=\frac{x_{i} v^{i}}{r v_{m v}^{0}},
$$

we prove the following lemma:

Lemma II.3. The spatial support of the massive particles is contained, at time $t$, in the ball of center at the origin and radius

$$
R_{0}+t\left(\frac{1+2 \epsilon}{1-2 \epsilon}\right)^{\frac{1}{2}} \frac{\left(\left(C \epsilon+\left(1+V_{0}^{2}\right)^{1 / 2}\right)^{2}-1\right)^{\frac{1}{2}}}{C \epsilon+\left(1+V_{0}^{2}\right)^{1 / 2}} .
$$

Proof.-We note that

$$
\frac{d|v|_{2}}{d t}=\frac{v_{i}}{|v|_{2}} \frac{\Gamma_{\alpha \beta}^{i}}{v_{m v}^{0}} v^{\alpha} v^{\beta} \leq \epsilon C(\epsilon) t^{-1-\alpha}\left(1+|v|_{2}^{2}\right)^{1 / 2} .
$$

By integration,

$$
\left(1+|v|_{2}^{2}\right)^{1 / 2} \leq C \epsilon+\left(1+V_{0}^{2}\right)^{1 / 2},
$$

for some constant $C$. Consider now the equation satisfied by massive particles: for $r=|x|_{2}$, we have

$$
\begin{aligned}
\left|\frac{d r}{d t}\right| & =\left|\frac{x_{i} v^{i}}{r v_{m v}^{0}}\right| \leq\left(\frac{1+2 \epsilon}{1-2 \epsilon}\right)^{\frac{1}{2}} \frac{|v|_{2}}{\left(1+|v|_{2}^{2}\right)^{1 / 2}} \\
& \leq\left(\frac{1+2 \epsilon}{1-2 \epsilon}\right)^{\frac{1}{2}} \frac{\left(\left(C \epsilon+\left(1+V_{0}^{2}\right)^{1 / 2}\right)^{2}-1\right)^{\frac{1}{2}}}{C \epsilon+\left(1+V_{0}^{2}\right)^{1 / 2}},
\end{aligned}
$$

which leads to the result.

Consider now massless particles. The following lemma holds. 
Lemma II.4. There exists a ball of radius $r(t)$ and centered at the origin such that the ball and the support of massless particles are disjoint. The radius of this ball obeys the differential inequality

$$
\begin{aligned}
\frac{d r_{m l}}{d t} \geq & \frac{1}{V_{0} e^{C \epsilon} \cdot\left(R_{0}+\left(\frac{1+2 \epsilon}{1-2 \epsilon}\right)^{1 / 2} t\right)} \\
& \cdot\left(-R_{0} V_{0}+t\left(\frac{1-2 \epsilon}{1+2 \epsilon}\right)^{1 / 2} V_{00} e^{-C \epsilon}(1-C \epsilon)\right) .
\end{aligned}
$$

Proof.-Since

$$
\left|\frac{d|v|_{2}}{d t}\right|=\left|\frac{v_{i}}{|v|_{2}} \frac{\Gamma_{\alpha \beta}^{i}}{v_{m l}^{0}} v^{\alpha} v^{\beta}\right| \leq C \epsilon t^{-1-\alpha}|v|_{2},
$$

we obtain immediately by integration,

$$
V_{00} e^{-C \epsilon} \leq|v|_{2} \leq V_{0} e^{C \epsilon} .
$$

Furthermore, since,

$$
\left|\frac{d r}{d t}\right|=\left|\frac{x_{i} v^{i}}{r v_{m l}^{0}}\right| \leq\left(\frac{1+2 \epsilon}{1-2 \epsilon}\right)^{1 / 2},
$$

we obtain

$$
r \leq R_{0}+\left(\frac{1+2 \epsilon}{1-2 \epsilon}\right)^{1 / 2} t
$$

Finally, differentiating $x_{i} v^{i}$, leads to

$$
\frac{d\left(x_{i} v^{i}\right)}{d t} \geq\left(\frac{1-2 \epsilon}{1+2 \epsilon}\right)^{1 / 2} V_{00} e^{-C \epsilon}(1-C \epsilon)
$$

and, $x_{i} v^{i}$ consequently satisfies

$$
x_{i} v^{i} \geq-R_{0} V_{0}+t\left(\frac{1-2 \epsilon}{1+2 \epsilon}\right)^{1 / 2} V_{00} e^{-C \epsilon}(1-C \epsilon)
$$

for some constant $C$. This quantity is positive for $t$ large enough. Plugging this inequality in the expression of the derivative of $r$, we obtain the result.

We want to realize the inequality, for $\epsilon$ small enough

$$
\begin{aligned}
K_{1} & :=\frac{1}{4} \frac{1}{V_{0} e^{C \epsilon} \cdot\left(\left(\frac{1+2 \epsilon}{1-2 \epsilon}\right)^{1 / 2}\right)} \cdot\left(\left(\frac{1-2 \epsilon}{1+2 \epsilon}\right)^{1 / 2} V_{00} e^{-C \epsilon}(1-C \epsilon)\right) \\
& \geq\left(\frac{1+2 \epsilon}{1-2 \epsilon}\right)^{\frac{1}{2}\left(\left(C \epsilon+\left(1+V_{0}^{2}\right)^{1 / 2}\right)^{2}-1\right)^{\frac{1}{2}}}=K_{2}
\end{aligned}
$$

At $\epsilon=0$, the inequality becomes

$$
\frac{1}{4} \frac{V_{00}}{V_{0}} \geq \frac{V_{0}}{\left(1+V_{0}^{2}\right)^{1 / 2}} .
$$

Take $V_{0}<1 / 16$, choose, $V_{00}=1 / 4 V_{0}$, then, the inequality above becomes

$$
\frac{1}{16} \geq \frac{V_{0}}{\left(1+V_{0}^{2}\right)^{1 / 2}},
$$

which is clearly realized since $V_{0}<1 / 16$. Hence, with this choice of $V_{0}$, there exists an $\epsilon$ small enough such that Eq. (4) is realized.

Now, since lim $\inf _{t \rightarrow \infty} d r / d t \geq 4 K_{1}$, there exists a time $t_{1}$ such that, for all $t>t_{1}$,

$$
\frac{d r}{d t} \geq 2 K_{1}
$$

Hence, by integration, for all $t>t_{1}$, we have

$$
r_{m l}(t) \geq 2 K_{1} t+r\left(t_{1}\right) .
$$

We already know that

$$
r_{m v}(t) \leq R_{0}+t K_{2}
$$

Since $K_{1} \geq K_{2}$, then for $t>t_{1}$

$$
r_{m l}(t) \geq 2 K_{1} t+r\left(t_{1}\right) \geq R_{0}+t K_{2} \geq r_{m v}(t) .
$$

Hence, we have proven:

Proposition II.5. For all $V_{0}<1 / 16$, for all $\epsilon$ small enough (depending on $V_{0}$ ), there exists $V_{00}$ (depending on $V_{0}$ ) and a time $t$ (depending on $\epsilon, V_{0}$ and $R_{0}$ ), and constant $C_{1}$ and $C_{2}$ (depending on all quantities above), such that

$$
\begin{gathered}
\operatorname{supp}_{x} f^{m v} \subset B\left(0, C_{1} t+C_{2}\right) \\
\text { and } \quad B\left(0, C_{1} t+C_{2}\right) \cap \operatorname{supp}_{x} f^{m l}=\varnothing .
\end{gathered}
$$

\section{Decomposition of the spacetime}

We detail in this section how the decomposition of the spacetime is performed. Firstly, using the local stability, we can evolve the solution up to a time $T$ satisfying the following requirements. At this time $t=T$, we can assume that the supports in space of the particles satisfy (see Sec. II B):

$$
\operatorname{supp}_{x} f^{m v} \subsetneq B\left(0, R_{1}\right) \text { and } B\left(0, R_{2}\right) \cap \operatorname{supp}_{x} f^{m l}=\varnothing,
$$

with $R_{1}<R_{2}$-see Fig. 2 for an illustration. It is important to remark that, by Birkhoff's theorem, in the region containing no matter, the metric coincides with the Schwarzschild solution. It is useful to describe the causal structure of this part to use the tortoise coordinate $r^{*}$, see cf. Chapter 5.5. of [13].

The past of the region $B\left(0, R_{1}\right)$ is called region $\mathrm{I}$. The past of $\left\{u=T-r^{*}\left(R_{1}\right)\right\}$ intersected with the complement of region I is called region II. In particular, the regions 


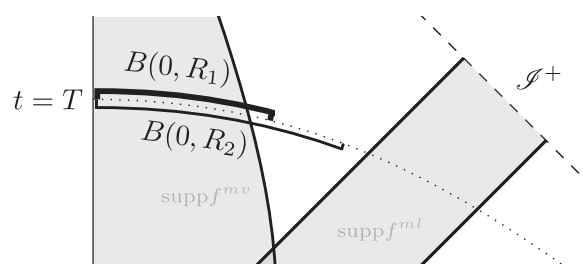

FIG. 2. On the slice $\{t=T\}$ all massive particles are contained in the ball $B\left(0, R_{1}\right)$ while the massless particles are outside of the ball $B\left(0, R_{2}\right)$, with $R_{1}<R_{2}$. As outlined in Sec. II C this is the starting point for decomposing the spacetime in regions I, II and III.

I and II are disjoint. The domain of influence $B\left(0, R_{2}\right)$ is called region III. In particular, the regions II and III are not disjoint, but, in their intersection, there is by construction no particle. Hence, in their intersection, the metric coincide with the Schwarzschild metric.

In region II, we use null coordinates similar to the one used by Dafermos. Since the massless particles travel at the speed of light, these particles remain confined in region II.

To analyze the solution in region III, consider the following construction. There exists a spacelike hypersurface $H$ satisfying the following conditions. In the intersection of regions III and II, this hypersuface is given by

$$
t^{2}-r_{*}^{2}=k^{2},
$$

for some constant $k$. Within the domain of influence of $B\left(0, R_{1}\right)$, this hypersurface extends smoothly as a compact set with boundary. The existence of such a parametrization in the complement of the domain of influence is discussed afterwards. By construction, the future of $H$ does not intersect the interior of region II.

\section{Global existence in region II}

In this section, we establish statements which are then applied to prove existence in region II, i.e., existence in a region bounded in $u$ near $\mathcal{I}^{+}$. The statements are similar to [5, Chapter 1, Theorem 1.1].

By [14] there exist null coordinates $(u, v, \theta, \varphi)$ which are convenient here, see [5]. The proof in [5] relies on the fact that the support of the massless distribution function remains compact in $u$, so the equations are integrated along $u$-slices inside the support. Since this is also given in the present case, the proof of existence up to some finite $u_{1}$ near $\mathcal{I}^{+}$does not change. We refer to [5] for details of the proof.

Denote by $T_{u u}, T_{u v}$ and $T_{v v}$ the projections of the energy momentum tensor onto the null directions respectively, see [5].

As illustrated in Fig. 3, we slice the region in the compact part of $v$-slices, bounded by a $u$-slice.

Let $u_{1}, v_{0}>0$ and for $v \geq v_{0}$ denote

$$
C_{v}:=\{v\} \cap\left\{u<u_{1}\right\}
$$

and the domain of influence of $C_{v_{0}}$ by $\mathcal{C}_{\infty}$.

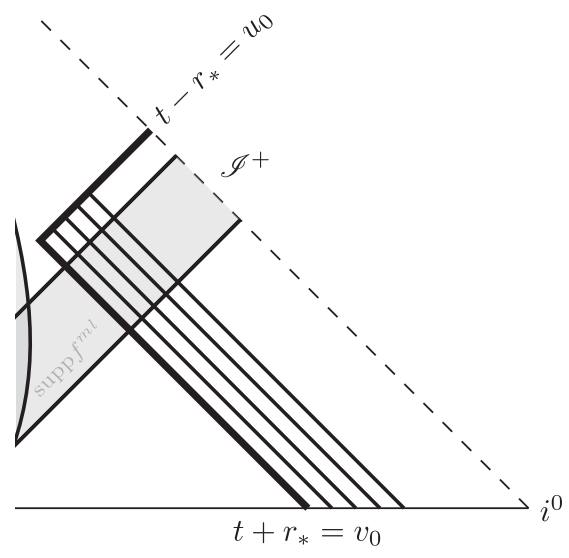

FIG. 3. No massive particles are in region II, whereas all the massless particles stay inside it. Outside the shaded region the spacetime is identical to Schwarzschild.

Now let $\left(f^{m v}, f^{m l}, \mu, \lambda\right)$ be a maximal solution of (EVS) on $\mathcal{C}_{\infty}$ with the massless support satisfying

$$
\left.\operatorname{supp} f^{m l}\right|_{\left\{v=v_{0}\right\} \times \mathbb{R}^{3}} \subset C_{v_{0}} \times B\left(0, p_{0}\right)^{c}
$$

for a $p_{0}>0$ and the spatial support of $f^{m v}$ and $\mathcal{C}_{\infty}$ being disjoint.

Theorem II.6 ([5, Theorem 1.1]). There exists $\epsilon$ small enough such that if the induced initial data satisfies

$$
\left\|f^{m l}\right\|_{L^{\infty}\left(C_{v_{0}}\right)} \leq \epsilon,
$$

then the solution exists globally on $\mathcal{C}_{\infty}$. Moreover the decay rates

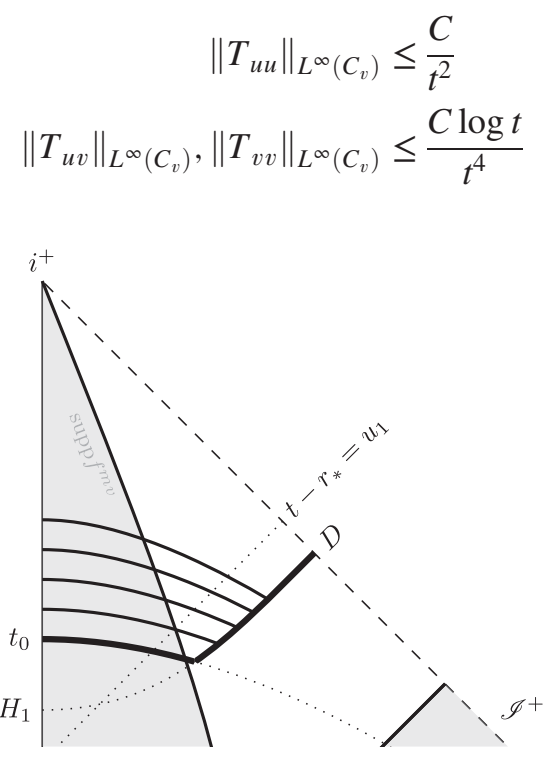

FIG. 4. The causal future of the union of the $t_{0}$-slice until vacuum and of $H_{1}$ in the vacuum region contains only massive particles. This region is then foliated by the compact part of $t$-slices. 
hold on $\mathcal{C}_{\infty}$ with some constant $C$. Moreover any outgoing null geodesic passing through $\mathcal{C}_{\infty}$ remains in $\mathcal{C}_{\infty}$ and is future complete [15].

Remark.-The proof of Theorem II.6 also provides that-due to initial compactness-the $v$-support of $f^{m l}$ is bounded from both sides by two $u$-slices inside $\mathcal{C}_{\infty}$.

\section{E. Global existence in region III}

We establish here existence in region III, where the separation of massive and massless particles has already occurred, and in that region only massive particles remain.

Consider the unit hyperboloid

$$
H_{1}=\left\{(t, x)\left|1=\sqrt{t^{2}-r_{*}(|x|)^{2}},\right| x \mid>2 M\right\},
$$

where $M$ is the ADM mass and $r_{*}(r)=r+2 M \log \left|\frac{r}{2 M}-1\right|$ is the tortoise coordinate for $r>2 M$, cf. [13, Chapter 5.5].

Let $r_{0}>2 M \geq 0, \quad w_{0} \geq 0, \quad t_{0}:=\sqrt{r_{*}\left(r_{0}\right)^{2}+1}$ and $\left(f^{m v}, f^{m l}, \mu, \lambda\right)$ be a maximal solution of (EVS) in the causal future of $D:=\Sigma_{0} \cup B$ where

$$
\Sigma_{0}:=\left\{t=t_{0},|x|<r_{0}\right\}, \quad B:=H_{1} \cap\left\{|x| \geq r_{0}\right\},
$$

with $f^{m v}$ compactly supported on $D \times \mathbb{R}^{3}$. Also assume that the spatial support of $f^{m v}$ intersected with $D$ is contained in $\Sigma_{0}$

$$
\left.\operatorname{supp} f^{m v}\right|_{D \times \mathbb{R}^{3}} \subset \Sigma_{0} \times B\left(0, w_{0}\right) \text {. }
$$

By construction there is vacuum in $B$, so we have Schwarzschild data for the metric coefficients $\mu$ and $\lambda$ there. So for $r \geq r_{0}>2 M r_{*}$ is invertible and we denote the inverse by $r_{*}^{-1}$. Then the radius on the hyperboloid as a function of $t$ is given by

$$
R_{0}(t):=r_{*}^{-1}\left(\sqrt{t^{2}-1}\right),
$$

and it follows that a point $(t, x) \in H_{1}$ is contained in $B$ if $t \geq t_{0}$.

$\mu$ and $\lambda$ are depending on radius and time only, so for $t \geq t_{0}$ and $|x| \geq r_{0}$ from the explicit form of the Schwarzschild metric we have

$$
\begin{aligned}
\mu\left(t, R_{0}(t)\right) & =\mu_{0}(t):=\frac{1}{2} \log \left(1-\frac{2 M}{R_{0}(t)}\right) \\
& =:-\lambda_{0}(t)=-\lambda\left(t, R_{0}(t)\right) .
\end{aligned}
$$

As we are interested in the future of $D$ which is for each time spatially compact, we foliate it by the compact part of $t$-slices for $t \geq t_{0}$

$$
\Sigma_{t}:=\{t\} \times B\left(0, R_{0}(t)\right) .
$$

The domain of influence of $D$ is denoted by $S_{\infty}$. In Fig. 4 the foliation used in region III is illustrated.

Proposition II.7. There exists $\epsilon$ small enough such that if the induced initial data satisfy

$$
\left\|f^{m v}\right\|_{L^{\infty}\left(\Sigma_{t_{0}}\right)} \leq \epsilon
$$

then the solution exists globally on $S_{\infty}$.

Moreover, the decay rates

$$
\begin{aligned}
\|\rho\|_{L^{\infty}\left(\Sigma_{t}\right)}, \quad\|p\|_{L^{\infty}\left(\Sigma_{t}\right)}, \quad\|j\|_{L^{\infty}\left(\Sigma_{t}\right)}, \quad\|q\|_{L^{\infty}\left(\Sigma_{t}\right)} & \leq \frac{C}{(1+t)^{3}} \\
\|\lambda\|_{L^{\infty}\left(\Sigma_{t}\right)}, \quad\|\mu\|_{L^{\infty}\left(\Sigma_{t}\right)} & \leq \frac{C}{1+t} \\
\left\|\Gamma_{\beta \gamma}^{\alpha}\right\|_{L^{\infty}\left(\Sigma_{t}\right)} & \leq \frac{C}{(1+t)^{2}} \\
\left\|R_{\beta \gamma \delta}^{\alpha}\right\|_{L^{\infty}\left(\Sigma_{t}\right)} & \leq \frac{C}{(1+t)^{3}}
\end{aligned}
$$

hold on $S_{\infty}$ with some constant $C$. Moreover any future directed causal geodesic passing through $S_{\infty}$ remains in $S_{\infty}$, and $S_{\infty}$ is future causally geodesically complete.

Remark.-Note that Proposition II.7 also holds true if the unit hyperboloid is shifted in $t$. In particular, replacing $H_{1}$ with

$$
H_{1, \tau}=\left\{(t, x) \mid 1=\sqrt{(t-\tau)^{2}-r_{*}(|x|)^{2}}\right\}
$$

for $\tau \geq 0$ and adapting $t_{0}=\sqrt{r_{*}\left(r_{0}\right)^{2}+1}+\tau$ and $R_{0}(t)=$ $r_{*}^{-1}\left(\sqrt{(t-\tau)^{2}-1}\right)$ accordingly.

The proof of the above statements can be carried out in a similar fashion to that of [1, Chapter 1.5, Theorem 5.1] in the massive only case, by using a bootstrap argument in the hyperboloidal foliation.

Since we work in the hyperboloidal foliation and the region is bounded by a part of a hyperboloid, when integrating the equations along $t$-slices additional boundary terms appear, in comparison with [1].

The proof of the decay of the matter fields given the decay of the geometric quantities is the same as [1, Chapter 1.5.1], since the way it is carried out relies solely on characteristics, which do not change because they just stay inside of the bounded region.

To prove the decay of the geometric quantities similar to [1, Chapter 1.5.2], one can see that $R_{0}$ is of order $t$, i.e., there exist constants $C_{1} \leq C_{2}$ such that

$$
C_{1} t \leq R_{0}(t) \leq C_{2} t .
$$


That estimate plays the role of $r_{0}+t$ in [1], which bounds $r$ in the nonvacuum region there.

In the following by $\tilde{C}$ we denote a constant which may depend on $C_{1}, C_{2}, r_{0}$, initial bounds on $\lambda$ and bounds on $\rho$ only.

Also since we have Schwarzschild data on $B$ with $\lambda_{0}(t)=-\mu_{0}(t)$

$$
\begin{aligned}
0 & \geq-\int_{r}^{R_{0}(t)}\left(\lambda^{\prime}(t, s)+\mu^{\prime}(t, s)\right) d s \\
& =-\lambda_{0}(t)-\mu_{0}(t)+\lambda(t, r)+\mu(t, r)=\lambda(t, r)+\mu(t, r)
\end{aligned}
$$

still holds.

For the expression of $\dot{\mu}$ the additional boundary terms appearing are the following

$$
4 \pi R_{0}(t) d_{2}\left(t, R_{0}(t)\right)+\frac{t}{R_{0}(t)} \cdot\left(\dot{\mu}\left(t, R_{0}(t)\right)-e^{2 \lambda\left(t, R_{0}(t)\right)}\left(-\frac{m\left(t, R_{0}(t)\right)}{R_{0}(t)^{2}}+4 \pi R_{0}(t) p\left(t, R_{0}(t)\right)\right)\right),
$$

where

$$
d_{2}(t, r):=e^{\mu+\lambda} \int\left(\frac{x \cdot \mathrm{v}}{r}\right)^{2} f \frac{d \mathrm{v}}{1+|\mathrm{v}|^{2}}
$$

and

$$
m(t, r)=4 \pi \int_{0}^{r} s^{2} \rho(t, s) d s
$$

Now $d_{2}$ can still be estimated by $\rho$ and for the first term in (10) we have

$$
R_{0}(t)\left|d_{2}\left(t, R_{0}(t)\right)\right| \leq R_{0}(t) \tilde{C} t^{-3} \leq \frac{\tilde{C}}{(1+t)^{2}}
$$

By (9) we have

$$
\begin{aligned}
\dot{\mu}\left(t, R_{0}(t)\right) & =\frac{t}{4 R_{0}(t)^{2}} \frac{1}{\frac{R_{0}(t)}{2 M}-1} \\
& \leq \tilde{C} \frac{1}{R_{0}(t)} \frac{1}{R_{0}(t)-2 M} \leq \frac{\tilde{C}}{(1+t)^{2}} .
\end{aligned}
$$

By the assumption on the initial data we have $p\left(t, R_{0}(t)\right)=0$ and $m\left(t, R_{0}(t)\right)$ is uniformly bounded. Summing that up the second term in (10) can also be estimated by $\frac{\tilde{C}}{t^{2}}$.

The final conclusion, to obtain global existence as stated in Proposition II.7, can be drawn completely analogously to [1, Chapter 1.5.3], while taking into account the additional boundary terms in the proof of the decay of $\lambda$ and $\mu$.

For $\lambda$ we have

$$
\lambda(t, r)=\lambda_{0}(t)-\int_{r}^{R_{0}(t)} e^{2 \lambda}\left(-\frac{m(t, s)}{s^{2}}+4 \pi \rho(t, s)\right) d s
$$

and since $\lambda$ is bounded and $\left|\lambda_{0}(t)\right| \leq M / R_{0}(t) \leq \tilde{C} /(1+t)$

$$
\begin{aligned}
|\lambda(t, r)| & \leq\left|\lambda_{0}(t)\right|+\tilde{C} \int_{r}^{R_{0}(t)} \frac{m(t, s)}{s^{2}} d s+\tilde{C} \int_{r}^{R_{0}(t)} \frac{1}{(1+t)^{3}} d s \\
& \leq\left|\lambda_{0}(t)\right|+\frac{\tilde{C}}{(1+t)}
\end{aligned}
$$

holds for $t \geq t_{0}, r \in\left[0, R_{0}(t)\right]$ and some constant $\tilde{C}$.

Those considerations work analogously for $\mu$ as the expression is similar and $\mu_{0}=-\lambda_{0}$. So finally we obtain

$$
\|\lambda\|_{L^{\infty}\left(\Sigma_{t}\right)}, \quad\|\mu\|_{L^{\infty}\left(\Sigma_{t}\right)} \leq \frac{C}{(1+t)} .
$$

Considering the geodesic equation and the decay rate $\left|\Gamma_{\beta \gamma}^{\alpha}\right| \lesssim(1+t)^{-2}$ we obtain the future geodesic completeness directly follows similar to [1, Chapter 1.5.3].

\section{F. Proof of Theorem I.1}

To prove the main theorem we combine Proposition II.1, Proposition II.5, Theorem II.6 and Proposition II.7.

We start with region I where due to Proposition I there exists a local in time solution of II.1 and Proposition II.5 provides a time slice which future is split into region II and region III. Then Theorem II.6 gives existence in region II, with properties of $f^{m l}$ such that we get existence in region III from Proposition II.7.

Choosing $\epsilon$ small enough the given initial data satisfy (1). So we use Proposition II.1 here to obtain a unique spherically symmetric solution $\left(f^{m v}, f^{m l}, \mu, \lambda\right)$ to (EVS) on the right maximal existence interval $[0, T)$ with $f^{m}(0)=f_{0}^{m}$. Here $T=T(\epsilon)>0$ as obtained from Proposition II.2. Again by requiring $\epsilon$ small, there is a $T^{\prime} \in$ $[0, T)$ such that Proposition II.5 holds. By Lemma II.3 and Lemma II.4 (5) in fact holds for all $t \in\left[T^{\prime}, T\right)$.

We now want to apply Theorem II.6. In order to do that first observe that, defining $\tilde{u}:=t-r_{*}(|x|)$ and $\tilde{v}:=$ $t+r_{*}(|x|)$ for $(t, x)$ in the vacuum region after the decomposition, then $(\tilde{u}, \tilde{v}, \theta, \varphi)$ are null coordinates in that region. Following Sec. III of [14], we know that for null coordinates $(u, v, \theta, \varphi)$ used in Sec. II D; in fact, for any other null coordinates considered in the vacuum region, 
there exist increasing functions $f$ and $g$ such that $u=f(\tilde{u})$ and $v=g(\tilde{v})$. Additionally, as $(\tilde{u}, \tilde{v}, \theta, \varphi)$ and $(u, v, \theta, \varphi)$ are equivalent by a diffeomorphism in that region, $f$ and $g$ are strictly increasing and bounded. So bounds in $\tilde{u}$ translate directly into bounds in $u$ and the same is true for $\tilde{v}$ and $v$.

For $R>0$ minimal such that $\operatorname{supp}_{x} f^{m v}\left(T^{\prime}\right) \subset B(0, R)$ and $t \geq T^{\prime}$ by Lemma II.3

$$
\operatorname{supp}_{x} f^{m v}(t) \subset B\left(0, R+\alpha\left(t-T^{\prime}\right)\right)
$$

holds for $\alpha>0$.

We choose $\tilde{u}_{0}=T^{\prime}-r_{*}(R)$ and $\tilde{v}_{0}=T^{\prime}+r_{*}(R)$. From Lemma (II.3) (6) holds. Also we parametrize the $(\tilde{u}, \tilde{v})$-boundary

$$
\begin{aligned}
& \tilde{u}_{B}(t):=t-r_{*}\left(R+\alpha\left(t-T^{\prime}\right)\right), \\
& \tilde{v}_{B}(t):=t+r_{*}\left(R+\alpha\left(t-T^{\prime}\right)\right) .
\end{aligned}
$$

of the support of the massive distribution function (11) by $t$.

Differentiating $\tilde{u}_{B}(t)$ w.r.t. $t$

$$
\frac{d \tilde{u}_{B}}{d t}=1-\alpha \frac{d r_{*}}{d r}\left(R+\alpha\left(t-T^{\prime}\right)\right)
$$

one can see that for $t>T^{\prime}$, by choosing the initial data small enough such that

$$
\alpha \leq 1-\frac{2 M}{R},
$$

$\frac{d \tilde{u}_{B}}{d t}>0$ is obtained. So for $t>T^{\prime}$ we have $\tilde{u}_{B}(t)>\tilde{u}_{0}$. That together with the fact that $\tilde{v}_{B}(t)<\tilde{v}_{0}$ for $t<T^{\prime}$ implies that the support of $f^{m v}$ and $\mathcal{C}_{\infty}$ are disjoint.

Proposition II.1 and Proposition II.5 imply existence of $\left(f^{m v}, f^{m l}, \mu, \lambda\right)$ in region I and existence in region II is implied by Theorem II.6.

It remains to prove existence in region III. In order to apply Proposition II.7 we first choose $\tilde{u}_{1}=\tilde{T}-r_{*}(\tilde{R})$ with $\tilde{T} \geq T^{\prime}$ and $\tilde{R}>2 M>0$ such that $\operatorname{supp}_{x} f^{m v}(\tilde{T}) \subset B(0, \tilde{R})$

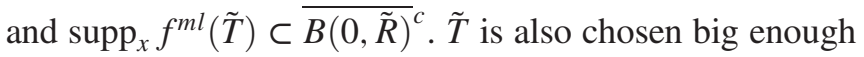
to ensure $\tilde{u}_{1}>\tilde{u}_{0}$. This choice is possible due to local stability. Next we choose $\tau=\frac{\tilde{u}_{1}+\tilde{u}_{0}}{2}$ and consider the in time shifted hyperboloid $H_{1, \tau}$. Finally let $\tilde{v}_{1}=\frac{1}{\tilde{u}_{1}-\tau}+\tau$.
With this construction we obtain that the part of the hyperboloid $H_{1, \tau}$ where $t+r_{*}(|x|)=\tilde{v} \geq \tilde{v}_{1}$ lies between the $\tilde{u}$-slices $\tilde{u}_{0}$ and $\tilde{u}_{1}$. So only a finite part of the hyperboloid is in region III, whereas the rest of it is in region II, where existence is already provided.

To see that this is true, consider $(t, r) \in H_{1, \tau}$ then

$$
\begin{gathered}
{\left[(t-\tau)-r_{*}(|x|)\right]\left[(t-\tau)+r_{*}(|x|)\right]} \\
\quad=(t-\tau)^{2}-r_{*}(|x|)^{2}=1 \geq 0
\end{gathered}
$$

and so

$$
\tilde{u}=t-r_{*}(|x|) \geq \tau \text {. }
$$

Similarly for $t+r_{*}(|x|)=\tilde{v} \geq \tilde{v}_{1}$

$$
\tilde{u}=t-r_{*}(|x|)=\frac{1}{t+r_{*}(|x|)-\tau}+\tau \leq \frac{1}{\tilde{v}_{1}-\tau}+\tau \leq \tilde{u}_{1} .
$$

Finally take $w_{0}=\sup \left\{\mid v \| v \in \operatorname{supp}_{v} f^{m v}\right\}$ and $r_{0}=$ $\delta+\sup \left\{|x| \mid(t, x, p) \in \operatorname{supp} f^{m v} \cap H_{1, \tau}\right\}$ for some $\delta>0$. We also know that $\operatorname{supp}_{x} f^{m l} \cap S_{\infty}=\varnothing$ from Theorem II.6 and the remark at the end of that section. This is all we need to get existence on $S_{\infty}$ due to Proposition II.7.

Considering this result together with the previously discussed existence in region II, this yields that $\left(f^{m v}, f^{m l}, \mu, \lambda\right)$ is a global in time solution.

The fact that the spacetime is future causally geodesically complete is a conclusion from the statements regarding causal geodesics in Theorem II.6 together with the remark thereafter, which implies that any future causal geodesic which is not outgoing ends up in region III, and Proposition II.7. The desired decay rate of the energy momentum tensor in region II is obtained from Theorem II.6 and the decay rate in region III is given by Proposition II.7.

\section{ACKNOWLEDGMENTS}

The authors are grateful to the anonymous referee for suggestions. This work has been supported by the Austrian Science Fund (FWF) project "Geometric transport equations and the non-vacuum Einstein flow" (P 29900-N27).
[1] G. Rein, The Vlasov-Einstein System with Surface Symmetry (Habilitation, Ludwig-Maximilians-Universität, Munich, 1995).

[2] See Sec. II C for a precise definition.

[3] See Secs. II D and II E for the definitions of $C_{v}$ and $\Sigma_{t}$. Also see Fig. 1 for a picture of the regions I, II, and III.
[4] G. Rein and A. D. Rendall, Commun. Math. Phys. 150, 561 (1992).

[5] M. Dafermos, J. Hyperbolic Differ. Equ. 3, 589 (2006).

[6] D. Fajman, J. Joudioux, and J. Smulevici, arXiv:1707 .06141 . 
[7] H. Lindblad and M. Taylor, arXiv:1707.06079.

[8] M. Taylor, Ann. PDE 3, 9 (2017).

[9] Y. Choquet-Bruhat, Ann. Inst. Fourier (Grenoble) 21, 181 (1971).

[10] H. Ringström, On the Topology and Future Stability of the Universe, Oxford Mathematical Monographs (Oxford University Press, New York, 2013).

[11] M. Taylor, Ann. PDE 3, 9 (2017).

[12] C. Svedberg, Future Stability of the Einstein-Maxwell-Scalar Field System and Non-linear Wave Equations Coupled to
Generalized Massive-Massless Vlasov Equations (KTH Royal Institute of Technology, Stockholm, 2012).

[13] S. W. Hawking and G. F. R. Ellis, The Large Scale Structure of Space-Time (Cambridge University Press, Cambridge, England, 2006).

[14] D. Christodoulou, Arch. Ration. Mech. Anal. 130, 343 (1995).

[15] By outgoing null geodesic, we denote a null geodesic for which $d v / d s>0$. Future complete then means that it exists for arbitrary positive $v$. 